\title{
Physiological Relevance of Angiotensin Converting Enzyme 2 As a Metabolic Linker and Therapeutic Implication of Mesenchymal Stem Cells in COVID-19 and Hypertension
}

\author{
Sarama Saha ${ }^{1}$. Sasanka Chakrabarti ${ }^{2} \cdot$ Praveen Kumar Singh $^{1} \cdot$ Jit Poddar $^{2} \cdot$ Soumitra Satapathi $^{3} \cdot$ Surendra Saini $^{4}$. \\ Sham S. Kakar ${ }^{5}$. Partha Roy ${ }^{4}$ \\ Published online: 3 August 2020 \\ (C) Springer Science+Business Media, LLC, part of Springer Nature 2020
}

\begin{abstract}
Severe acute respiratory syndrome corona virus -2 (SARS-CoV-2) is a single stranded RNA virus and responsible for infecting human being. In many cases the individual may remain asymptomatic. Some recently reported studies revealed that individuals of elderly age group and with pre-existing medical conditions such as hypertension, diabetes mellitus had severe consequences, even may lead to death. However, it is not clearly delineated whether hypertension itself or associated comorbidities or antihypertensive therapy contributes to the grave prognosis of COVID-19 infections. This review is aimed to decipher the exact mechanisms involved at molecular level from existing evidence and as reported. It has been reported that SARS-CoV-2 enters into the host cell through interaction between conserved residues of viral spike protein and angiotensin converting enzyme 2 (ACE2) receptor which is highly expressed in host's cardiac and pulmonary cells and finally transmembrane protease, serine-2 (TMPRSS2), helps in priming of the surface protein. Subsequently, symptom related to multi organ involvement is primarily contributed by cytokine storm. Although various clinical trials are being conducted on renin- angiotensin- system inhibitor, till to date there is no standard treatment protocol approved for critically ill COVID-19 positive cases with pre-existing hypertension. Recently, several studies are carried out to document the safety and efficacy outcome of mesenchymal stem cell transplantation based on its immunomodulatory and regenerative properties. Therefore, identification of future novel therapeutics in the form of mesenchymal stem cell either alone or in combination with pharmacological approach could be recommended for combating SARS-CoV-2 which might be dreadful to debilitating elderly people.
\end{abstract}

Keywords Angiotensin converting enzyme 2 . Renin-angiotensin-aldosterone-system - Severe acute respiratory syndrome . Coronavirus-2 $\cdot$ Spike protein $\cdot$ COVID-19 $\cdot$ Transmembrane protease serine-2, Mesenchymal Stem Cell

This article belongs to the Topical Collection: Special Issue on COVID19 Pandemic and Stem Cells

Guest Editor: Mariusz Z. Ratajczak

Partha Roy

patha.roy@bt.iitr.ac.in

1 Department of Biochemistry, All India Institute of Medical Sciences, Rishikesh India

2 Department of Biochemistry and Central Research Cell, Maharishi Markandeshwar (deemed to be) University, Mullana Haryana India

3 Department of Physics, Indian Institute of Technology Roorkee, Roorkee Uttarakhand 247667 India

4 Department of Biotechnology, Indian Institute of Technology Roorkee, Roorkee Uttarakhand 247667 India

5 Department of Physiology and James Graham Brown Cancer Center, University of Louisville, Louisville KY 40292 USA

\section{Epidemiology of COVID 19}

A novel corona virus which is responsible for ongoing outbreak of fatal acute respiratory tract infection was named as SARS-CoV-2 (Severe Acute Respiratory syndrome associated coronavirus) by International Committee on Taxonomy of Viruses (ICTV). This virus emerged from Wuhan, China, in December 2019. The disease was named by WHO on 11th of February 2020 as coronavirus disease 2019 (COVID-19) [1]. The regional epidemic then spread to all over the world. On 12th of March, 2020, WHO declared it as pandemic. According to Worldometer (URL:https://www.worldometers.info/ coronavirus/), as of 4th June 2020, globally total infected cases were $6,596,713$ while $3,188,018$ cases recovered and this contagious disease caused death of 388,424 individuals. However, COVID-19 has shown very 
dissimilar outcome in terms of severity and mortality in different nations which are not easily explained [2]. Human corona virus (CoV) was first reported in 1965 and belongs to the family coronaviridae. Based on phylogeny, $\mathrm{CoV}$ are divided into four genera: alpha, beta, gamma and delta. Only beta $\mathrm{CoV}$ genus contains four lineages $\mathrm{A}$, $\mathrm{B}, \mathrm{C}$ and D (Fig. 1). All identified human corona viruses belong to either alpha or beta genera and gamma and delta primarily include avian origin. New SARS COV 2 is a beta coronavirus from the subgenus Sarbecovirus [3].

The current 2019 novel coronavirus is a single stranded RNA virus which contains various structural and nonstructural proteins. Following entry through respiratory tract, spike (S) protein of coronavirus binds to the angiotensin converting enzyme related carboxypeptidase (ACE2) host receptor for its entry into the host cell. The structural proteins of SARS-COV-2 are shown in Fig. 2.

\section{Physiological Relevance of ACE2}

ACE2, a monocarboxypeptidase glycoprotein, is constitutively expressed in various organs such as lungs, gastrointestinal tract, kidney, endothelium, heart and to some extent in pancreas. It has two forms: (i) the full-length membrane bound ACE2 which has an extracellular domain acting as a receptor for the spike protein of SARSCOV-2, transmembrane domain and cytoplasmic domain which contains calmodulin binding site [4]; (ii) soluble form of ACE2 which circulates in the blood [5]. On the other hand, Angiotensin converting enzyme (ACE) is a zinc dependent dipeptidyl carboxypeptidase having two catalytic homologous domains that plays an important role in the regulation of blood pressure [6]. Although there is approximately $60 \%$ sequence similarity with the catalytic domain of ACE, the ACE inhibitors (ACEi) such
Fig. 1 Classification with examples of common coronaviruses and presenting symptoms

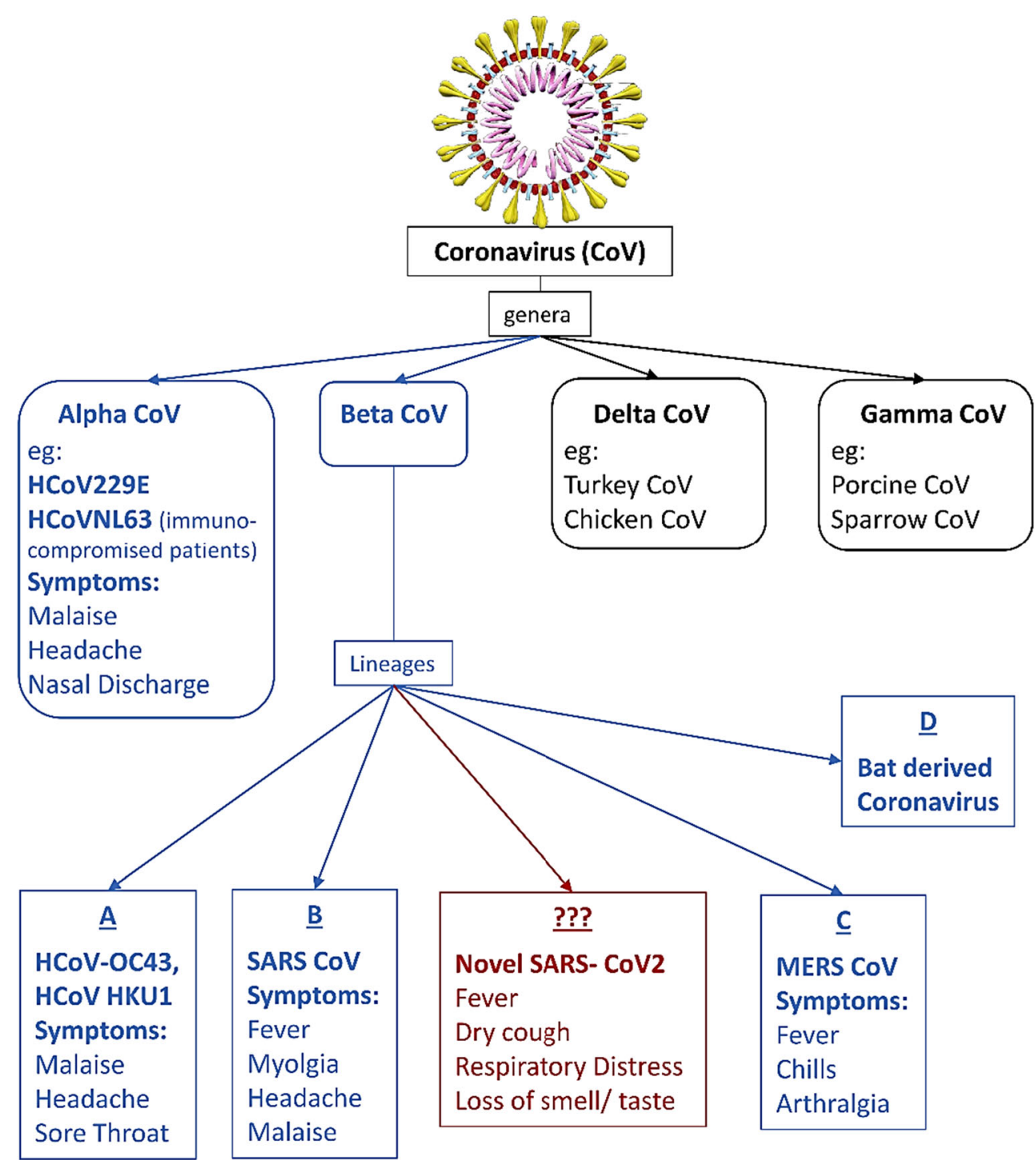




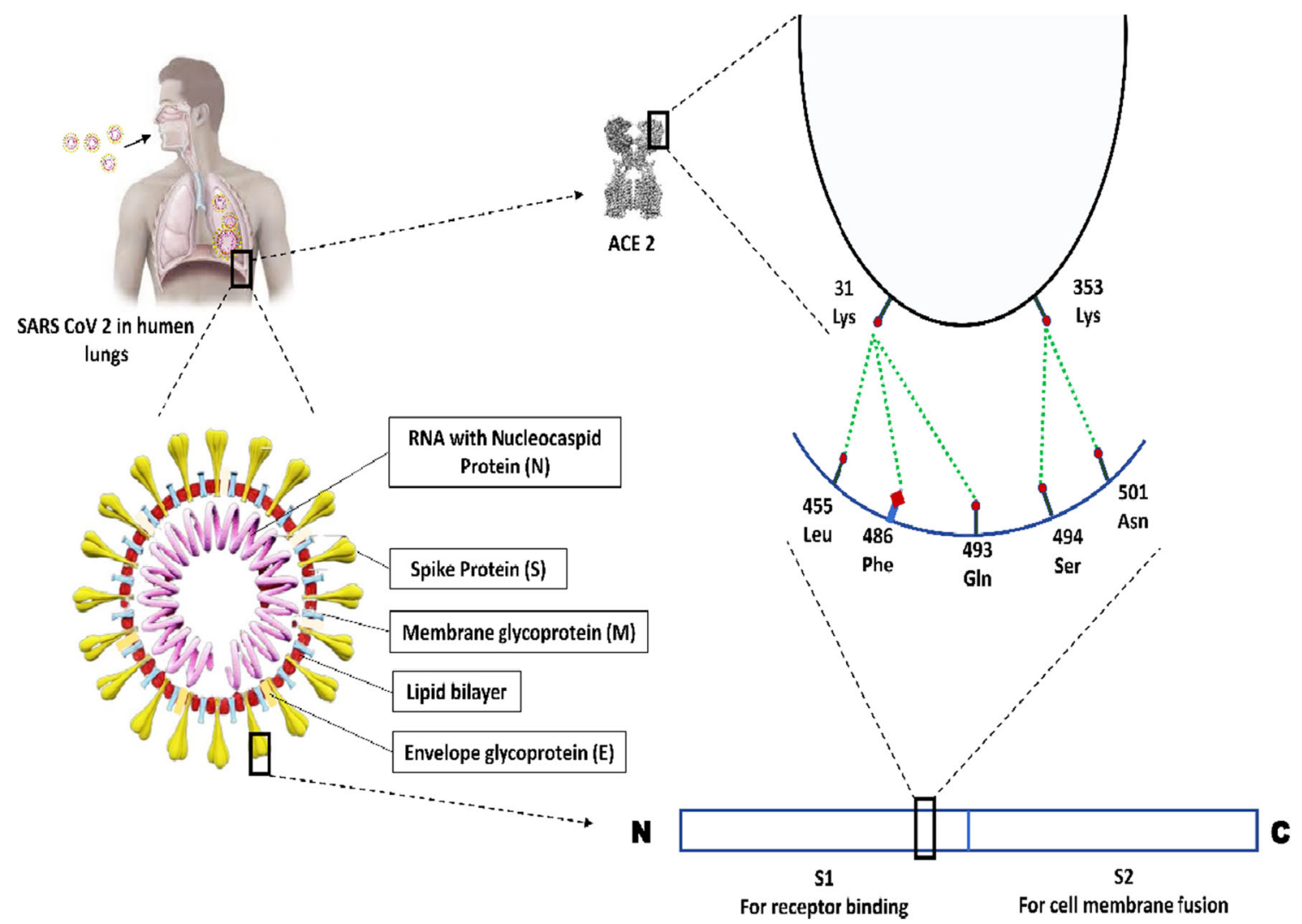

Fig. 2 Schematic diagram depicting the conserved residues for interaction between viral S protein and host receptor ACE2. Leu, leucine; Phe, phenylalanine; Gln, glutamine, Asn, asparagine; Lys, lysine; Ser, serine

as Lisiniopril, Ramipril do not attenuate the effect of ACE2 since the active sites differ [7]. Hence ACEi does not seem to have direct effect on ACE2. However, it can indirectly increase the expression of ACE2 to meet the increased demand of metabolism of accumulated Angiotensin (Ang) I and Ang II. The mechanism involved in the metabolism of Ang I and II has been presented in Fig. 3. The protective effect of ACE2 on cardiovascular system is mediated through Ang(1-7) which is the main degradation product of Ang II. Catalytic as well as noncatalytic role of ACE2 in the metabolic process is depicted in Fig. 3. Transmembrane protease, serine 2 (TMPRSS2) present in host cell helps in facilitating the entry of virus through priming of the Surface (S) protein which is functionally divided into two parts i.e., S1 and $\mathrm{S} 2$ domains [8]. The $\mathrm{S} 1$ domain is responsible for receptor binding (RBD) and S2 domain contributes to cell membrane fusion. Thus, the transmissibility of COVID-19 is primarily determined by specific interaction between conserved residues located on RBD of S protein and ACE2 receptor of host cell [3]. Interaction between virus and the host through specific residues has been schematically depicted in Fig. 2. Because of these conserved residues, the COVID-19 is considered to have capacity of transmission from human to human.

\section{Clinical Evidence}

The novel coronavirus infection commonly presents with respiratory symptoms such as dry cough, pneumonia and in some cases, rapidly progresses to respiratory distress requiring admission in the intensive care unit (ICU). Few recent studies conducted during the outbreak in China reported that approximately $20-30 \%$ of patients were vulnerable to develop severe consequences having distinctive co-morbidities such as diabetes mellitus (DM), hypertension, chronic obstructive pulmonary disease, cardiovascular and cerebrovascular diseases [11-13]. Guan et al. [12] documented that 15\% of total 1099 COVID patient had hypertension as co-morbidity. Bhandari et al. [14] conducted a study on 21 COVID positive patients in North India, and reported that approximately $14 \%$ of participants had pre-existing hypertension and $10 \%$ had DM. Prevalence rates of co-morbidities in 8 recent studies [11-18] conducted in 2020 are presented in Table 1. Patients with distinct coexisting diseases were found to have significantly higher death rate [for hypertension odds ratio (OR), 3.05; confidence interval (CI), 1.57 to $5.92 ; \mathrm{p}<0.006$; for DM, OR, 2.85; CI, 1.4-6.1; $<<0.001$; for CVD, OR, 21.4; CI, 4.7-98.8; p < 0.0001], indicating that comorbidities are potential determinant of morbidity and mortality of COVID-19 infection [19]. However, some patients 


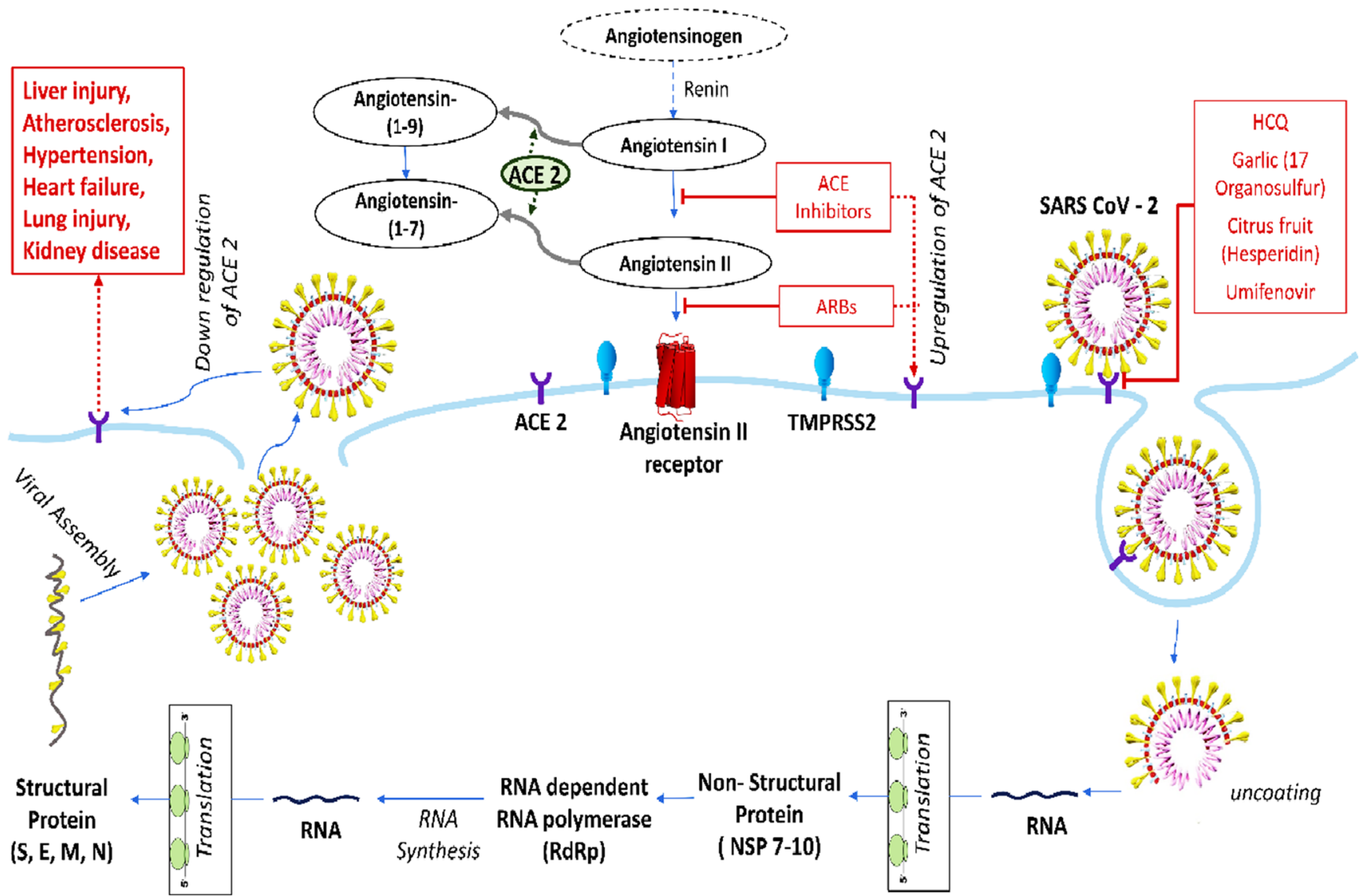

Fig. 3 Schematic presentation of the replication of virus and mechanism of action of ACE2 in host cell (alveolar cell) [9, 10]. TMPRSS2, transmembrane protease, serine 2; HCQ, hydroxy chloroquine; ARB,

angiotensin II receptor blocker; S, spike protein; E, envelope glycoprotein; M, membrane glycoprotein; N, nucleocapsid protein

with co-morbidities had initial atypical presentation. In a recent case study, it was reported that a 62 years old hypertensive and pre-diabetic female presented with dysarthria and right extremity weakness. She had very high level of Ferritin, D- Dimer, C reactive protein (CRP) and lactate dehydrogenase (LDH). Following treatment with intravenous thrombolysis, limb movement was improved [20]. Avula et al. [21] reported 4 cases of age range 73-88 years for the development of altered mental status, limb weakness and slurred speech. The blood level of diagnostic parameters such as D-Dimer, Ferritin and CRP of these patients had direct relation to the clinical consequences and mortality. Abdulkadir et al. [22] conducted a study on 4 cases mostly aged over 60 years and having a pre-existing hypertension and or DM presented with features suggestive of stroke which was confirmed by the presence of infarct in large or small cerebral vessels in cerebral imaging. They also had elevated levels of Ferritin and D- Dimer. Even after treatment with aspirin and low molecular weight heparin, patients did not show much improvement. From these studies it is obvious that most of the patients who were vulnerable to develop severe illness in the form of admission in ICU, required mechanical ventilation, or death as one of the outcomes for elderly age groups with distinctive co-morbidities having hypertension as common co-morbidity.

The most obvious and intriguing issue that needs to be addressed is, why advancing age makes the person vulnerable to COVID-19 and is associated with poor prognosis? In this regards, multiple explanations could be put forward.

1. It has been reported that ACE2 expression is increased in elderly people [23], in heart failure and arterial hypertension [24] and thus predispose them to get infected with SARS-CoV-2.

2. With the advancing age the immune system in human goes through the phase of immunosenescence resulting in depletion of adaptive immunity and achievement of nonspecific innate immunity. Moreover, disintegration of regulatory $\mathrm{T}$ cells (Treg) cell function and attenuation of immunoinhibitory skills will allow the amplification of pathogens and make the older individual prone to get infected [25].

3. Most of the old people are found to be associated with various medical conditions either alone or in combination with DM, hypertension, cardiovascular disease. 


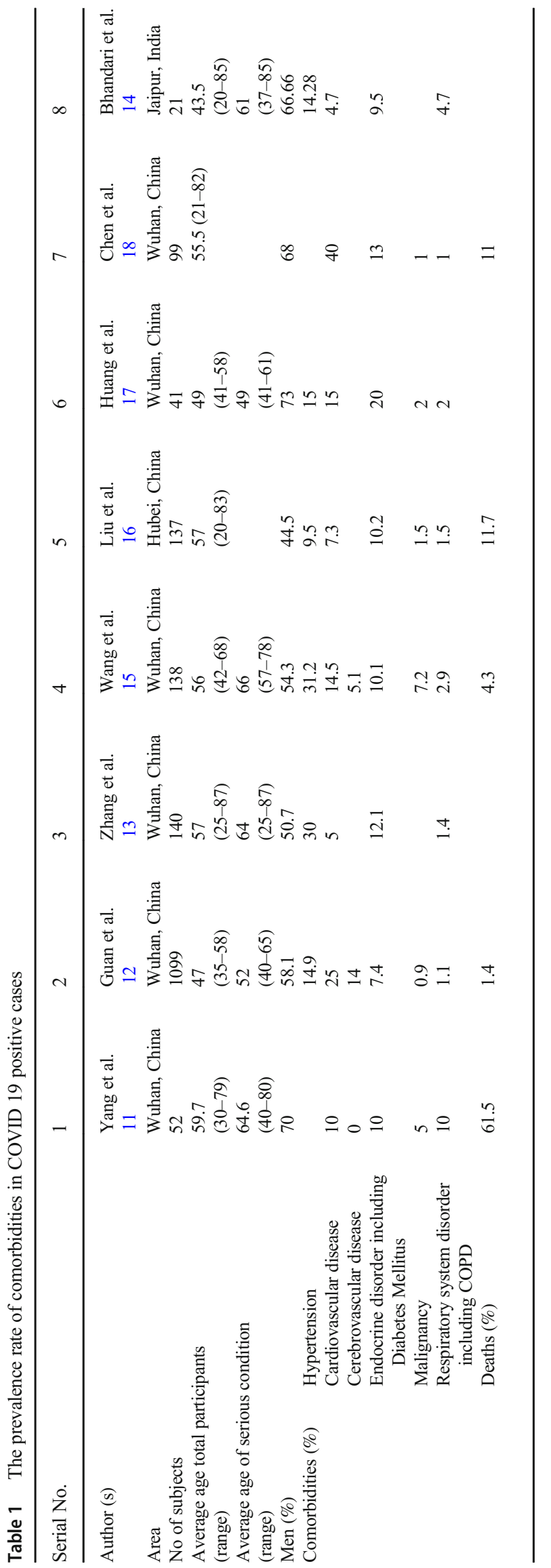

Interestingly, the second largest population of patients with DM lives in India (International Diabetes Federation 2019). It has been reported that uncontrolled glycemia compromises the front-line defence, the innate immune system, towards SARS-COV-2 infection resulting in uncontrolled proliferation of infectious agents. Moreover, aggravated response of proinflammatory cytokines such as IL-1, IL-6 and TNF- $\alpha$ associated with hyperglycemia in patients with COVID19 exacerbate the symptoms of respiratory distress resulting in fatal outcome [26]. Furthermore, with increase in duration of DM as well as in some cases, old people with hypertension need treatment with ACEi which may increase the expression of ACE2.

4. Moreover, Pioglitazone and Liraglutide used for controlling the blood glucose level have been found to upregulate the expression of ACE2 in experimental model [27, 28].

Increased expression of ACE2 as well as low immunity enhances the binding of virus to the host receptors and makes the elderly aged people more susceptible to COVID-19 infection. In-spite of having protective role of ACE2 to the heart and increased expression of ACE2 following treatment with ACEi, the COVID-19 patients had poor prognosis. It may be explained by the fact that viral intrusion subsequently causes downregulation of ACE2 expression resulting in over activation of Renin-Angiotensin-Aldosterone-System (RAAS). The uncontrolled aggravation of aldosterone level may lead to loss of water and electrolyte homeostasis which will attenuate the protective impact of ACE2 and leads to various organ injury such as lung, heart, liver, pancreas which will present as multiorgan failure.

\section{Biochemical Correlation With Severity of SARS-CoV-2 Infection}

Biochemical investigation reported a decrease in hemoglobin count whereas a significant increase in Ferritin, CRP and lactate dehydrogenase [20-22]. The non-structural proteins of coronavirus such as $1 \mathrm{ab}, \mathrm{ORF} 10$ and ORF $3 \mathrm{a}$ dissociates iron from hemoglobin giving rise to porphyrin. Subsequently ORF 8 and surface glycoproteins form a complex with porphyrin and thus interfere with the synthesis of hemoglobin by reducing the availability of heme. The decrease in hemoglobin content will lead to attenuation of oxygen exchange at alveolar level. In order to store the large amount of released iron, there will be rise in the level of ferritin [29].

In severely ill patients blood investigation reports revealed a significantly higher level of D-dimer, prolonged activated partial thromboplastin time and low platelet count. This indicates that SARS-COV-2 may induce disseminated intravascular coagulation resulting in thromboembolism in pulmonary 
blood vessel giving rise to the symptoms of respiratory distress and features suggestive of small and large vessels infarction. This could be explained by the decrease in ACE2 expression following coronavirus infection resulting in attenuation of the ACE2/ angiotensin-(1-7)/ Mas receptor axis and prevention of protection against thrombosis [30]. This probable mechanism has been depicted in Fig. 3 .

\section{Comparison With Impact of Co-morbidities in Middle East Respiratory Syndrome (MERS) and Severe Acute Respiratory Syndrome (SARS)}

Badawi and Ryoo [31] conducted a systematic analysis on 637 MERS-CoV positive cases and reported that approximately half of these populations had co-morbidities such as DM and hypertension who had a dreadful clinical outcome and even death accounting to $35 \%$ of positive cases. Kulcsar et al. [32] developed a diabetic mice model which was prone to get infected with MERS-CoV. Following infection, the mice had severe consequences and delayed recovery compared to normal mice. Diabetic mice had very few inflammatory cells as well as decreasing trend of TNF- $\alpha$, IL 6 and IL12b, suggestive of abnormal immune responses. From this study, authors inferred that dysregulation of immune responses might contribute to the disease severity in MERS $\mathrm{CoV}$ positive cases with co-morbidities. Yang et al. [33] conducted a study on 539 SARS positive cases with history of $\mathrm{DM}$ and concluded that diabetes is an independent determinant of morbidity and mortality of SARS patients.

Surprisingly, most of the studies did not provide any details of treatment for co-morbidities of COVID-19 affected individuals. Considering the world-wide use of RAAS inhibitors, we have discussed in this review about the impact of ACEi/ Angiotensin II receptor blocker (ARB) on COVID-19 infection and the interplay between ACE2 receptors and SARSCoV-2 infection.

\section{Contradictory Evidence Related to ACEi and ARB}

The crucial effect of ACEi on cardiovascular system is produced by inhibiting the conversion of Ang I to Ang II resulting in decrease in plasma Ang II and attenuating the hydrolytic degradation of bradykinin. ACEi exhibits its vasodilatory and fibrinolytic effects through increase in blood level of bradykinin, prostaglandin and nitric oxide [34, 35]. Moreover, ACEi had been documented to produce its anti-inflammatory, antifibrotic, antithrombotic and vasodilatory effects through the activation of ACE2/ Ang(1-7)/ MAS axis [36]. The cardioprotective role of this axis has been documented in various in vivo animal and in vitro cell culture models. It was reported that either administration of Losartan alone or in combination with Lisinopril, could enhance the expression of mRNA and the activity of ACE2 in rat cardiac membrane [37]. Several investigations had been carried out to translate the experimental results into clinical prosperity. ACEi increases the expression of ACE2 as an acquired response to counter-balance the enhanced levels of Ang I and II. Since ACE2 is the most important and well identified host receptor for the entry of virus into host cell, the aggravated expression might increase the risk of COVID-19 infection.

On the contrary, one retrospective study conducted by Henry et al. [38] revealed that patients who were on ACEi treatment required less endotracheal intubation during severe respiratory tract infection and thus reduced the chances of mortality. Moreover, Losartan showed a significant beneficial effect in acute lung injury caused by acid aspiration [39] as well as in experimental model of ACE2 knockout mice [40] and mice infected with coronavirus [41]. Furthermore, it was documented that ARB therapy could significantly decrease the risk of death in Ebola patients [42].

\section{Clinical Trial on ACEi/ ARB and Human Recombinant ACE2}

It is evident that ACE2 has contribution both in the pathogenesis of hypertension and acute respiratory syndrome caused by SARS-CoV-2. From this fact it could be speculated that SARS-CoV-2 not only involves pulmonary system but could also affect other ACE2 expressing organs such as cardiovascular systems and cerebrovascular system. Moreover, individuals with pre-existing hypertension and were treated with ACEi and or ARB might have influenced the prognosis of COVID-19 induced respiratory symptoms. Previous studies showed contradictory findings related to the impact of ACEi and ARBs on human and experimental model. Hence various clinical trials had been conducted throughout the world to observe the role of these inhibitors on modulation of the severity and prognosis of individuals with SARS-COV-2 infection.

A Single-Center Retrospective Observational case control study was conducted in Wuhan, China between March 21-30, 2020 (https://clinicaltrials.gov/ct2/show/NCT04318301). In this study a total of 275 participants were divided into two groups. Group 1 included COVID-19 positive patients with hypertension receiving $\mathrm{ACEi} / \mathrm{ARB}$ for the treatment of hypertension and Group 2 included COVID-19 positive patients with hypertension but not receiving ACEi/ARB. A randomized controlled trial on Losartan (https://clinicaltrials.gov/ct2/ show/NCT04312009) is also being carried out by University of Minnesota, USA from April 13, 2020. In this study 200 COVID-19 positive hospitalized patients were enrolled. They 
were placed in two arms. The participants of experimental and placebo comparator arms received Losartan $50 \mathrm{mg}$ and placebo (microcrystalline methylcellulose, gelatine capsule) respectively daily. A similar randomized control trial of Losartan (25 mg daily) is being conducted on 580 COVID19 positive patients who do not require hospitalization (https:// clinicaltrials.gov/ct2/show/NCT04311177). Another randomized clinical trial has been launched by National University of Ireland, Galway, Ireland on March 30, 2020. In this study 2414 hypertensive COVID-19 positive patients were recruited. The experimental arm participants of this study were switched to alternative medication for hypertension such as calcium channel blocker/ diuretics and the active comparator arm would continue treatment with ACEi/ ARB. Aim of this study is to determine whether continuation of ACEi/ ARB will influence the risk of death among COVID19 positive individuals. A randomized interventional clinical trial sponsored by Assistance Publique - Hôpitaux de Paris has been started since April 92020 (https://clinicaltrials.gov/ct2/ show/NCT04329195). Aim of this study is to evaluate if RAS blockers should be continued in SARS-CoV-2 infected individuals with history of cardiovascular disease.

Two interventional clinical trials (NCT04375046 and NCT04382950) are going to be conducted by Kafrelsheikh University from May 2020 to determine whether alone Recombinant Bacterial ACE2 Receptors - Like Enzyme of B38-CAP or in combination with Aerosolized 13 cis-retinoic acid would be more effective than Recombinant Human ACE2 to control lung injury in COVID-19 positive patients. An interventional two arm pilot study (NCT04287686) had been launched by Guangzhou Medical University to determine the effect of recombinant human ACE2 in adult COVID-19 patients since February 2020. However, it has been withdrawn without $\mathrm{CDE}$ approval. Recruitment of one clinical trial (NCT04318301) has been completed. Otherwise, to the best of our knowledge, in most of the cases recruitment is still going on. A pilot clinical trial (NCT01597635) was conducted on 44 acute lung injury patients to evaluate the safety and tolerability of recombinant human ACE2. At the end of phase II trial it was found to be safe in subjects with acute respiratory distress syndrome [43].

\section{ACE2 as Therapeutic Target}

ACE2 along with TMPRSS2 play an important role in entry of virus into the host cell. These could be targeted as a prevention of initiation and spread of viral infection. Hydroxycholoroquine which is commonly used for the treatment of malaria may be repurposed for preventing the entry of virus by inhibiting the glycosylation of host receptor and thus inhibiting the binding of spike protein of the virus to the active site of host receptor [19]. Moreover, Umifenovir, an established drug for treatment and prophylaxis of influenza, is a promising repurposed drug for its action to interfere the interplay between $\mathrm{S}$ protein and ACE2 for SARS-CoV-2 viral entry as evidenced by in vitro studies [44, 45].

Camostatmesylate, an approved drug in Japan for the treatment of pancreatitis, showed a great hope by in vitro study with its inhibitory effect against host serine protease TMPRSS2 which helps in S protein priming to interact with ACE2 [45] and thus prevents entry of the virus into the host cell. Moreover, it has some regulatory effect on blood lipid and glucose level [46]. Therefore, it might be considered superior to ACE2 blocker since it will interfere with the entry of viral pathogen but at the same time preserve the protective effect of ACE2 especially in COVID-19 patients with dyslipidemia and/ or hyperglycemia. According to a recent study, 17 organosulfur compounds identified in garlic essential oil were considered to be valuable natural antiviral compound source that prevents the intrusion of coronavirus into the host cell by inhibiting interaction between host receptor ACE2 and the main protease PDB6LU7 of SARS-CoV2 [47].

Hesperidin is a bioflavonoid, a type of plant pigment with antioxidant and anti-inflammatory effects found primarily in unripe citrus fruit such as oranges, grapefruit, and lemon. The binding interface between Spike and ACE2 is targeted by Hesperidin to prevent entry of virus into the host cells [48]. It has been reported to have significant cardio-protective action as well [49].

Some health systems have suggested discontinuing the use of ACEi and ARB for prophylaxis as well as treatment of hypertension with respect to suspected infection during COVID-19 pandemic. However, it is too early to support or refute the claims based on limited data on the effective use of these drugs on COVID-19 positive individuals.

\section{Therapeutic Importance of ACE2 and Mesenchymal Stem Cell (MSC)}

Increasing rate of infection and subsequent complications and even mortality prompted researchers from various biomedical fields to find out solutions/ specific treatments in order to control this pandemic. However, to date there is no approved specific treatment targeting SARS-CoV-2 virus, other than some symptomatic treatment protocol such as hydration, supplementation of oxygen and mechanical ventilation. The characteristic pathogenic feature of COVID-19 is cytokine storm which includes the release of interleukin (IL)-2, IL-6, IL-7, and TNF- $\alpha$ [50]. These released cytokines are primarily responsible for endothelial cell damage leading to leakage of fluid, pulmonary edema, cardiac damage, kidney injury and finally multi organ failure. Recently, Ratajczak and Kucia [51] have documented the link between ACE2 and Nlrp3 inflammasome in initiating the cytokine storm. Following 
binding of SARS-CoV-2 to ACE2, it triggers the downstream signalling molecule resulting in activation of Nlrp3 inflammasome which can initiate immune response and cause cell death through activation of caspase 1. Furthermore, Nlrp3 could play an important role in perturbation of coagulation cascade by activation of complement cascade (ComC) giving rise to thrombotic manifestation in SARS-CoV-2 infected individuals. Ulrich and Pillat [52] provided an insight into the second entry point (CD147) for SARS-CoV-2. The expression of CD147 has been documented in mesenchymal stem cells (MSCs) of human cord blood. Ling et al. [53] documented the expression of ACE2 in pulmonary residing stem cells and progenitor cells in Oct-4 positive neonatal mice. The lack of these MSC like cells may be responsible for delaying in recovery from lung injury resulting in severe outcome in patients with SARS-CoV-2 infection compared to healthy individuals. Hence any cell based therapy which can modulate immune system and helps in regeneration would be an ideal method to treat this infection. Because of immunomodulatory and regenerative properties, MSCs has drawn attention to researchers from medical fraternity. Moreover, these cells have gained popularity recently because of following reasons: MSCs (i) can be isolated from easily available resources such as cord blood, dental pulp, menstrual blood, peripheral blood, adult adipose tissue and buccal pad of fat; (ii) has potentiality to differentiate; (iii) requires less invasive procedure for administration; (iv) can be stored for future use and (v) free of ethical issues [54, 55]. Various clinical trials on MSCs for the treatment of type 2 diabetes and autoimmune diseases have already established its safety and efficacy [56, 57]. However, to the best of our knowledge, MSC therapy for the treatment of COVID-19 patients has not yet been approved. In order to document its safety and effectiveness, various clinical trials are being conducted. Liang et al. [58] reported the outcome of a case study of human umbilical cord stem cell transplantation on 65 year old critically ill COVID-19 positive patient. Considering the improvement in biochemical parameters (such as serum bilirubin, CRP and ALT/ AST) along with some vital signs, it was opined that MSC could be considered as an ideal choice for the treatment of severe complications of SARS-CoV-2 infection. Leng et al. [59] conducted a study in China between January 23-31, 2020, where seven COVID-19 positive patients with different disease severity and medical history of hypertension for approximately 10 years had been enrolled. The aim of this study was to identify the safety and efficacy outcome of MSC transplantation in these patients. Following intravenous injection of MSCs, there was a significant decline in pro-inflammatory cytokines such as TNF- $\alpha$ and elevation of anti-inflammatory cytokines such as IL-10 and chemokines like interferon- $\gamma$ inducible protein (IP-10). After 13 days of post transplantation, the nucleic acid test was found to be negative. It also reversed the lymphopenia and CRP values which is a marker of myocardial damage.
RNA sequence analysis of transplanted MSCs confirmed the immunomodulatory property of MSCs because of expression of anti-inflammatory factors such as vascular endothelial growth factor and epidermal growth factor in MSCs and their ability to differentiate into AT2 (Angiotensin II receptor type 2) cells due to expression of surfactant proteins $A$ and $C$. These studies indicated that MSC either alone or in combination with other pharmacological drugs could be recommended as a therapeutic intervention for the improvement of outcome of COVID-19 patients after further detailed patient sample analysis.

\section{Mesenchymal Stem Cells and Cytokine Storm in COVID 19}

Previous studies conducted on SARS-CoV-2 patients reported a significant increase in certain circulating chemokines (CXCL8, CCL2 and CXCL10) and inflammatory cytokines (IL-1, IL-6 and IL-12). CXCL2 was considered as a useful prognostic marker for assessing the progress of SARS disease [60]. The cumulating epidemiological evidence suggest that approximately $80 \%$ of COVID-19 positive cases remain mild or asymptomatic and 5-6\% become critically ill thus progressing to acute respiratory distress syndrome and multiorgan failure [19]. In these cases, the human corona virus become highly pathogenic. The virus triggers acute release of cytokines such as IL-2, IL-6, TNF $\alpha$, granulocyte colony stimulating factors which in turn induces acute injury to multiple organs [50]. The injury to lung causes pulmonary oedema resulting in difficulty in air exchange at alveolar epithelium finally leading to acute respiratory distress syndrome which is typically identified by ground glass like appearance in X-ray. Similarly, patients may develop features with acute cardiac, liver and kidney injury. Recently available treatment modalities such as non-specific anti-viral therapy, corticosteroids and broad spectrum antibiotics for secondary bacterial infections could not control these severe conditions presented with cytokine release syndrome resulting in death of these critically ill patients. Recent reports on COVID-19 suggest that IL-6 pays the crucial role and its elevated circulating level has significant correlation with the severity of respiratory failure, ARDS, and adverse clinical outcomes [50]. In order to reduce the mortality, a few studies attempted to address these cases with MSC transplantation. However, the mode of actions of these MSCs is yet to be unveiled [58, 59].

Cumulating evidence revealed that adult MSCs can influence the response of immune cells through various soluble factors such as transforming growth factor $\beta 1$ (TGF $\beta 1$ ), prostaglandin E 2 (PGE2), nitric oxide (NO), anti-inflammatory cytokine IL-10 and indole amine -pyrrole 2,3 dioxygenase (IDO) [61]. At the same time MSCs can attenuate the T cell proliferation by secreting PGE2, NO and IDO and thus inhibit 
cytokine secretion (IFN- $\gamma$ and TNF- $\alpha$ ) and mitigate cytotoxicity $[62,63]$. Moreover, MSCs hinder the release of inflammatory cytokines like IL-17 and 22 through decrease in differentiation of naïve T cells to Th17 cells. Furthermore, they can increase the synthesis of anti-inflammatory cytokine IL-10 through modulation of transcription factor, fork head box P 3 and epigenetic changes [64]. This is possible because MSCs share various adhesion molecules such as vascular cell adhesion molecule, leucocyte function associated antigen 3 and intercellular adhesion molecule 1 with T cells. They can induce the Treg by cell-cell contact which is one of the crucial factors involved in the immunomodulatory properties of MSCs [65]. MSCs can reduce the proliferation of B cells and thus reduce the production of antibodies through involvement of TGF- $\beta$ and PGE2 [66]. Likewise, MSCs can reduce cytotoxicity by influencing cytokine release (IFN- $\gamma$ ) from NK cells through modulation of its proliferation which was induced by IL-2 and IL-15 [67]. Although there are innumerable soluble factors, the efficacy of these soluble factors on influencing the activity of MSCs depends on the sites from where the MSCs were isolated and the region where they would be engrafted. Administration of MSCs in experimental model of immune thrombocytopenia [68] and proctitis induced by radiation [69] reported that MSCs could improve the condition through reduction and increase in the production of inflammatory and anti-inflammatory cytokines respectively. Various clinical trials were conducted on patients with systemic lupus erythematosus (SLE), DM, ulcerative colitis and multiple sclerosis and they all have showed some promising outcomes in terms of patient recovery [70-72]. Lack of adequate scientific data, large variability in trial size as well as diversity in the origin of MSCs have brought about major limitations to exactly delineate the therapeutic benefit of stem cells for severe COVID-19 cases.

Synthetic stem cells "LIFNano" which was developed by involving cutting technologies of nanotechnology was found to be far more superior (almost 1000-fold) in terms of potency than soluble leukaemia inhibitory factor which is a fundamental requirement to control the cytokine storm induced by corona virus in critically ill patients. In experimental model of multiple sclerosis, LIFNano showed improvement in 4 days [50]. Considering the time line this could be useful for treatment of pneumonia in COVID-19 cases.

These characteristic features of MSCs prompted the researchers to consider creatively the use of these cells for the treatment of COVID-19 patients with severe manifestations. One clinical trial (NCT04437823) is being conducted by Johns Hopkins University where recruitment of patients has been started since June 2020. The main goal of this study is to investigate the efficacy of intravenous infusions of stem cells in the treatment of COVID-19 patients. As of 7th July 2020, almost 53 interventional phase I and/ or II clinical trials have been registered all over the world to observe the safety and efficacy of MSCs for the treatment of COVID 19 and/ or Pneumonia/ARDS patients (https://clinicaltrials.gov/ct2/ show/NCT04349631 ?term=mesenchymal+and+ covid\&draw). In these studies, MSCs were either derived from adipose tissues or from umbilical cords depending on respective cases. Although most of the studies adopted intravenous mode of administration, one pilot study (NCT04276987) is being conducted on aerosol inhalation of the exosomes derived from allogenic adipose MSCs for the treatment of serious COVID-19 pneumonia patients [73]. In almost all trials, recruitment of study participants is going on. The number of enrolled participants in these studies seems to be variable like in certain cases is $20-30$ and in others it is 90 100 . The results of these ongoing clinical trials may pave the way for confirmation of safety and potentiality of new stem cell based biological approach to reduce the severity of COVID-19.

\section{Future Prospects}

Further studies on coronavirus will be required to identify the proteins (structural and non-structural) other than ACE2 which are essential for interaction between virus and host cells to prevent the spread of infection. This information in future will help in the advancement of our knowledge about the cure and management of this virus infection in various ways. Firstly, this will help in understanding the proclivity of viruses towards specific type of host and thus provide information on early prediction of potential outbreaks. Secondly, the study on structural details would provide relevant novel therapeutic target to encounter infection at the early stage and prevent the progression into a pandemic infection. Thirdly, knowledge on immune-pathologic response of the host to viral proteins may help in developing vaccines to decrease the disease load. Taken together, all these aspects, there is an optimistic approach ahead which will help in the development of a novel drug for the treatment and management of SARS-CoV-2. It is presumed that these effective drug molecules could be either from natural sources or has to be synthesized based on the phytochemical-inspired structures. In addition to these aspects, the use of stem cell based therapies also appears to be a critical approach for the cure and management of this pandemic situation. However, this aspect needs further detailed analysis involving a large number of patients with special emphasis on age-dependent impacts. A large cohort study using MSCs will provide the exact therapeutic potentiality of this stem cell based approach. If this comes out to be successful, that will open up a complete new regime for the cure and management of SARS-CoV-2. The other alternative of novel therapeutics would be repurposing of drugs with better efficacy either alone or in combination with other drugs. All these 
aspects have to be considered together towards the development of strategies for combating SARS-CoV-2.

\section{Conclusion}

Based on the findings presented in this review article, it is evident that although COVID-19 is not principally a metabolic or endocrine disorder, optimum regulation of plasma glucose and blood pressure are essential to avoid the obvious threat and enhanced risk of development of relevant complications such as heart failure and stroke in patients with COVID-19. In order to avoid the adverse prognosis of COVID-19 infections, various public health measures should be taken into consideration. In this management preparedness protocol, policy makers should provide particular attention to those individuals who are elderly as well as having comorbidities such as DM and hypertension. Considering the lack of specific antiviral treatment for COVID-19 cases, in order to reduce the mortality, stem cell based therapy could be a novel therapeutic approach to decrease the severity of COVID-19 patients. Therefore, this aspect needs serious research and development where the existing data will provide a basis of taking up those in-depth studies. It is speculated that a combined approach as depicted in this review will probably help in the cure and management of elderly patients with associated co-morbidities.

\section{Compliance with Ethical Standards}

Conflict of Interest All authors have read the final version of the manuscript and have agreed to the content. Authors declare no conflict of interest.

\section{References}

1. Zhou, P., Yang, X. L., Wang, X. G., Hu, B., Zhang, L., Zhang, W., Si, H. R., Zhu, Y., Li, B., Huang, C. L., \& Chen, H.D. A pneumonia outbreak associated with a new coronavirus of probable bat origin. Nature, 579(7798), 270-273.

2. Chakrabarti, S. S., Kaur, U., Banerjee, A., Ganguly, U., Banerjee, T., Saha, S., et al. (2020). COVID-19 in India: Are biological and environmental factors helping to stem the incidence and severity? Aging and Disease, 11(3), 480-488.

3. Lu, R., Zhao, X., Li, J., Niu, P., Yang, B., Wu, H., Wang, W., Song, H., Huang, B., Zhu, N., \& Bi, Y. (2020). Genomic characterisation and epidemiology of 2019 novel coronavirus: implications for virus origins and receptor binding. The Lancet, 395(10224), 565-574.

4. Lambert, D. W., Clarke, N. E., Hooper, N. M., \& Turner, A. J. (2008). Calmodulin interacts with angiotensin-converting enzyme-2 (ACE2) and inhibits shedding of its ectodomain. FEBS Letters, 582(2), 385-390.

5. Batlle, D., Wysocki, J., \& Satchell, K. (2020). Soluble angiotensinconverting enzyme 2: a potential approach for coronavirus infection therapy? Clinical Science, 134(5), 543-545.
6. Bernstein, K. E., Khan, Z., Giani, J. F., Cao, D. Y., Bernstein, E. A., \& Shen, X. Z. (2018). Angiotensin-converting enzyme in innate and adaptive immunity. Nature Reviews Nephrology, 14(5), 325336.

7. Tikellis, C., \& Thomas, M. C. (2012). Angiotensin-converting enzyme 2 (ACE2) is a key modulator of the renin angiotensin system in health and disease. International Journal of Peptides, 2012(256294), 1-8.

8. Hoffmann, M., Kleine-Weber, H., Schroeder, S., Krüger, N., Herrler, T., Erichsen, S., et al. (2020). SARS-CoV-2 cell entry depends on ACE2 and TMPRSS2 and is blocked by a clinically proven protease inhibitor. Cell, 181(2), 271-280.e8.

9. Sanders, J. M., Monogue, M. L., Jodlowski, T. Z., \& Cutrell, J. B. (2020). Pharmacologic treatments for coronavirus disease 2019 (COVID-19): a review. JAMA, 323(18), 1824-1836.

10. Vaduganathan, M., Vardeny, O., Michel, T., McMurray, J. J., Pfeffer, M. A., \& Solomon, S. D. (2020). Renin-angiotensin-aldosterone system inhibitors in patients with Covid-19. New England Journal of Medicine, 382(17), 1653-1659.

11. Yang, X., Yu, Y., Xu, J., Shu, H., Liu, H., Wu, Y., Zhang, L., Yu, Z., Fang, M., Yu, T., \& Wang, Y. (2020). Clinical course and outcomes of critically ill patients with SARS-CoV-2 pneumonia in Wuhan, China: a single-centered, retrospective, observational study. The Lancet Respiratory Medicine, 8(5), 475-481.

12. Guan, W. J., Ni, Z. Y., Hu, Y., Liang, W. H., Ou, C. Q., He, J. X., et al. (2020). Clinical characteristics of coronavirus disease 2019 in China. New England Journal of Medicine, 382(18), 1708-1720.

13. Zhang, J. J., Dong, X., Cao, Y. Y., Yuan, Y. D., Yang, Y. B., Yan, Y. Q., Akdis, C. A., \& Gao, Y. D. (2020). Clinical characteristics of 140 patients infected with SARS-CoV-2 in Wuhan, China. Allergy, 75(7), 1730-1741.

14. Bhandari, S., Bhargava, A., Sharma, S., Keshwani, P., Sharma, R., $\&$ Banerjee, S. (2020). Clinical profile of covid-19 infected patients admitted in a tertiary care hospital in north India. The Journal of the Association of Physicians of India, 68, 13-17.

15. Wang, D., Hu, B., Hu, C., Zhu, F., Liu, X., Zhang, J., Wang, B., Xiang, H., Cheng, Z., Xiong, Y., \& Zhao, Y. (2020). Clinical characteristics of 138 hospitalized patients with 2019 novel coronavirus-infected pneumonia in Wuhan, China. Jama, 323(11), 1061-1069.

16. Liu, K., Fang, Y. Y., Deng, Y., Liu, W., Wang, M. F., Ma, J. P., et al. (2020). Clinical characteristics of novel coronavirus cases in tertiary hospitals in Hubei Province. Chinese Medical Journal, 133(9), 1025-1031.

17. Huang, C., Wang, Y., Li, X., Ren, L., Zhao, J., Hu, Y., Zhang, L., Fan, G., Xu, J., Gu, X., \& Cheng, Z. (2020). Clinical features of patients infected with 2019 novel coronavirus in Wuhan, China. The Lancet, 395(10223), 497-506.

18. Chen, N., Zhou, M., Dong, X., Qu, J., Gong, F., Han, Y., Qiu, Y., Wang, J., Liu, Y., Wei, Y., \& Yu, T. (2020). Epidemiological and clinical characteristics of 99 cases of 2019 novel coronavirus pneumonia in Wuhan, China: a descriptive study. The Lancet, 395(10223), 507-513.

19. Zhou, F., Yu, T., Du, R., Fan, G., Liu, Y., Liu, Z., Xiang, J., Wang, Y., Song, B., Gu, X., \& Guan, L. (2020). Clinical course and risk factors for mortality of adult inpatients with COVID-19 in Wuhan, China: a retrospective cohort study. The Lancet, 395(10229), 1054 1062.

20. Co, C. O. C., Yu, J. R. T., Laxamana, L. C., \& David-Ona, D. I. A. (2020). Intravenous thrombolysis for stroke in a COVID-19 positive Filipino patient, a case report. Journal of Clinical Neuroscience, 77, 234-236.

21. Avula, A., Nalleballe, K., Narula, N., Sapozhnikov, S., Dandu, V., Toom, S., Glaser, A., \& Elsayegh, D. (2020). COVID-19 presenting as stroke. Brain, Behavior, and Immunity, 87, 115-119. 
22. Abdulkadir, T. U. N.Ç, ÜNLÜBAȘ, Y., ALEMDAR, M., \& AKYÜZ, E. (2020). Coexistence of COVID-19 and acute ischemic stroke report of four cases. Journal of Clinical Neuroscience, 77, 227-229.

23. Bukowska, A., Spiller, L., Wolke, C., Lendeckel, U., Weinert, S., Hoffmann, J., Bornfleth, P., Kutschka, I., Gardemann, A., Isermann, B., \& Goette, A. (2017). Protective regulation of the $\mathrm{ACE} 2 / \mathrm{ACE}$ gene expression by estrogen in human atrial tissue from elderly men. Experimental Biology and Medicine, 242(14), $1412-1423$.

24. te Riet, L., van Esch, J. H., Roks, A. J., van den Meiracker, A. H., \& Danser, A. J. (2015). Hypertension: renin-angiotensin-aldosterone system alterations. Circulation Research, 116(6), 960-975.

25. Weyand, C. M., \& Goronzy, J. J. (2016). Aging of the immune system: Mechanisms and therapeutic targets. Annals of the American Thoracic Society, 13(Supplement 5), S422-S428.

26. Geerlings, S. E., \& Hoepelman, A. I. (1999). Immune dysfunction in patients with diabetes mellitus (DM). FEMS Immunology \& Medical Microbiology, 26(3-4), 259-265.

27. Fang, L., Karakiulakis, G., \& Roth, M. (2020). Are patients with hypertension and diabetes mellitus at increased risk for COVID-19 infection? The Lancet. Respiratory Medicine, 8(4), e21.

28. Romaní-Pérez, M., Outeiriño-Iglesias, V., Moya, C. M., Santisteban, P., González-Matías, L. C., Vigo, E., \& Mallo, F. (2015). Activation of the GLP-1 receptor by liraglutide increases ACE2 expression, reversing right ventricle hypertrophy, and improving the production of SP-A and SP-B in the lungs of type 1 diabetes rats. Endocrinology, 156(10), 3559-3569.

29. Liu, W., \& Li, H. (2020). COVID-19: attacks the 1-beta chain of hemoglobin and captures the porphyrin to inhibit human heme metabolism. Preprint revised on 12.07.2020.

30. Jiang, T., Gao, L., Lu, J., \& Zhang, Y. D. (2013). ACE2-Ang-(17)-Mas axis in brain: a potential target for prevention and treatment of ischemic stroke. Current Neuropharmacology, 11(2), 209-217.

31. Badawi, A., \& Ryoo, S. G. (2016). Prevalence of comorbidities in the Middle East respiratory syndrome coronavirus (MERS-CoV): a systematic review and meta-analysis. International Journal of Infectious Diseases, 49, 129-133.

32. Kulcsar, K. A., Coleman, C. M., Beck, S. E., \& Frieman, M. B. (2019). Comorbid diabetes results in immune dysregulation and enhanced disease severity following MERS-CoV infection. JCI insight, 4(20), e13774.

33. Yang, J. K., Feng, Y., Yuan, M. Y., Yuan, S. Y., Fu, H. J., Wu, B. Y., Sun, G. Z., Yang, G. R., Zhang, X. L., Wang, L., \& Xu, X. (2006). Plasma glucose levels and diabetes are independent predictors for mortality and morbidity in patients with SARS. Diabetic Medicine, 23(6), 623-628.

34. Iyer, S. N., Yamada, K., Diz, D. I., Ferrario, C. M., \& Chappell, M. C. (2000). Evidence that prostaglandins mediate the antihypertensive actions of angiotensin-(1-7) during chronic blockade of the renin-angiotensin system. Journal of Cardiovascular Pharmacology, 36(1), 109-117.

35. Li, P., Chappell, M. C., Ferrario, C. M., \& Brosnihan, K. B. (1997). Angiotensin-(1-7) augments bradykinin-induced vasodilation by competing with ACE and releasing nitric oxide. Hypertension, 29(1), 394-398.

36. Ferrario, C. M., Trask, A. J., \& Jessup, J. A. (2005). Advances in biochemical and functional roles of angiotensin-converting enzyme 2 and angiotensin-(1-7) in regulation of cardiovascular function. American Journal of Physiology-Heart and Circulatory Physiology, 289(6), H2281-H2290.

37. Ferrario, C. M., Jessup, J., Chappell, M. C., Averill, D. B., Brosnihan, K. B., Tallant, E. A., Diz, D. I., \& Gallagher, P. E. (2005). Effect of angiotensin-converting enzyme inhibition and angiotensin II receptor blockers on cardiac angiotensin-converting enzyme 2. Circulation, 111(20), 2605-2610.
38. Henry, C., Zaizafoun, M., Stock, E., Ghamande, S., Arroliga, A. C., \& White, H. D. (2018). Impact of angiotensin-converting enzyme inhibitors and statins on viral pneumonia. In Baylor University Medical Center Proceedings, Abingdon: Taylor \& Francis, 31(4) 419-423.

39. Kuba, K., Imai, Y., Rao, S., Gao, H., Guo, F., Guan, B., Huan, Y., Yang, P., Zhang, Y., Deng, W., \& Bao, L. (2005). A crucial role of angiotensin converting enzyme 2 (ACE2) in SARS coronavirusinduced lung injury. Nature Medicine, 11(8), 875-879.

40. Imai, Y., Kuba, K., Rao, S., Huan, Y., Guo, F., Guan, B., Yang, P., Sarao, R., Wada, T., Leong-Poi, H., \& Crackower, M. A. (2005). Angiotensin-converting enzyme 2 protects from severe acute lung failure. Nature, 436(7047), 112-116.

41. Yang, P., Gu, H., Zhao, Z., Wang, W., Cao, B., Lai, C., Yang, X., Zhang, L., Duan, Y., Zhang, S., Chen, W., Zhen, W., Cai, M., Penninger, J. M., Jiang, C., \& Wang, X. (2014). Angiotensinconverting enzyme 2 (ACE2) mediates influenza H7N9 virusinduced acute lung injury. Scientific Reports, 4(7027), 1-6.

42. Fedson, D. S., Jacobson, J. R., Rordam, O. M., \& Opal, S. M. (2015). Treating the host response to Ebola virus disease with generic statins and angiotensin receptor blockers. MBio, 6(3).

43. Khan, A., Benthin, C., Zeno, B., Albertson, T. E., Boyd, J., Christie, J. D., Hall, R., Poirier, G., Ronco, J. J., Tidswell, M., \& Hardes, K. (2017). A pilot clinical trial of recombinant human angiotensinconverting enzyme 2 in acute respiratory distress syndrome. Critical care, 21(1), 1-9.

44. Kadam, R. U., \& Wilson, I. A. (2017). Structural basis of influenza virus fusion inhibition by the antiviral drug Arbidol. Proceedings of the National Academy of Sciences, 114(2), 206-214.

45. Khamitov, R. A., Loginova, S., Shchukina, V. N., Borisevich, S. V., Maksimov, V. A., \& Shuster, A. M. (2008). Antiviral activity of arbidol and its derivatives against the pathogen of severe acute respiratory syndrome in the cell cultures. Voprosy Virusologii, 53(4), 9-13.

46. Jia, D., Taguchi, M., \& Otsuki, M. (2005). Synthetic protease inhibitor camostat prevents and reverses dyslipidemia, insulin secretory defects, and histological abnormalities of the pancreas in genetically obese and diabetic rats. Metabolism, 54(5), 619-627.

47. Thuy, B. T. P., My, T. T. A., Hai, N. T. T., Hieu, L. T., Hoa, T. T., Thi Phuong Loan, H., Triet, N. T., Anh, T. T. V., Quy, P. T., Tat, P. V., \& Hue, N. V. (2020). Investigation into SARS-CoV-2 resistance of compounds in garlic essential oil. ACS Omega, 5(14), $8312-8320$

48. Wu, C., Liu, Y., Yang, Y., Zhang, P., Zhong, W., Wang, Y., Wang, Q., Xu, Y., Li, M., Li, X., \& Zheng, M. (2020). Analysis of therapeutic targets for SARS-CoV-2 and discovery of potential drugs by computational methods. Acta Pharmaceutica Sinica B, 10(5), 766788.

49. Sobenin, I. A., Andrianova, I. V., Demidova, O. N., Gorchakova, T., \& Orekhov, A. N. (2008). Lipid-lowering effects of timereleased garlic powder tablets in double-blinded placebo-controlled randomized study. Journal of Atherosclerosis and Thrombosis, 15(6), 334-338.

50. Metcalfe, S. M. (2020). Mesenchymal stem cells and management of COVID-19 pneumonia. Medicine in Drug Discovery, 5, 100019.

51. Ratajczak, M. Z., \& Kucia, M. (2020). SARS-CoV-2 infection and overactivation of Nlrp3 inflammasome as a trigger of cytokine "storm" and risk factor for damage of hematopoietic stem cells. Leukemia, 34, 1726-1729.

52. Ulrich, H., \& Pillat, M. M. (2020). CD147 as a target for COVID19 treatment: suggested effects of azithromycin and stem cell engagement. Stem Cell Reviews and Reports. https://doi.org/10.1007/ s12015-020-09976-7.

53. Ling, T. Y., Kuo, M. D., Li, C. L., Alice, L. Y., Huang, Y. H., Wu, T. J., Lin, Y. C., Chen, S. H., \& Yu, J. (2006). Identification of pulmonary Oct- $4+$ stem/progenitor cells and demonstration of their 
susceptibility to SARS coronavirus (SARS-CoV) infection in vitro. Proceedings of the National Academy of Sciences, 103(25), 9530 9535.

54. Golchin, A., Farahany, T. Z., Khojasteh, A., Soleimanifar, F., \& Ardeshirylajimi, A. (2019). The clinical trials of mesenchymal stem cell therapy in skin diseases: an update and concise review. Current Stem Cell Research \& Therapy, 14(1), 22-33.

55. Golchin, A., Seyedjafari, E., \& Ardeshirylajimi, A. (2020). Mesenchymal stem cell therapy for COVID-19: present or future. Stem Cell Reviews and Reports. https://doi.org/10.1007/s12015020-09973-w.

56. Golchin, A., Shams, F., \& Karami, F. (2019). Advancing Mesenchymal stem cell therapy with CRISPR/Cas9 for clinical trial studies. Advances in Experimental Medicine \& Biology, 1247, 89-100.

57. Novello, S., Debouche, A., Philippe, M., Naudet, F., \& Jeanne, S. (2020). Clinical application of mesenchymal stem cells in periodontal regeneration: A systematic review and meta-analysis. Journal of Periodontal Research, 55(1), 1-12.

58. Liang, B., Chen, J., Li, T., Wu, H., Yang, W., Li, Y., Li, J., Yu, C., Nie, F., Ma, Z., \& Yang, M. (2020). Clinical remission of a critically ill COVID-19 patient treated by human umbilical cord mesenchymal stem cells. ChinaXiv, 2, v10.

59. Leng, Z., Zhu, R., Hou, W., Feng, Y., Yang, Y., Han, Q., Shan, G., Meng, F., Du, D., Wang, S., \& Fan, J. (2020). Transplantation of ACE2-mesenchymal stem cells improves the outcome of patients with COVID-19 pneumonia. Aging and Disease, 11(2), 216-228.

60. Wong, C. K., Lam, C. W. K., Wu, A. K. L., Ip, W. K., Lee, N. L. S., Chan, I. H. S., Lit, L. C. W., Hui, D. S. C., Chan, M. H. M., Chung, S. S. C., \& Sung, J. J. Y. (2004). Plasma inflammatory cytokines and chemokines in severe acute respiratory syndrome. Clinical \& Experimental Immunology, 136(1), 95-103.

61. Gao, F., Chiu, S. M., Motan, D. A. L., Zhang, Z., Chen, L., Ji, H. L., Tse, H. F., Fu, Q. L., \& Lian, Q. (2016). Mesenchymal stem cells and immunomodulation: current status and future prospects. Cell Death \& Disease, 7(1), e2062-e2062.

62. Ren, G., Zhang, L., Zhao, X., Xu, G., Zhang, Y., Roberts, A. I., Zhao, R. C., \& Shi, Y. (2008). Mesenchymal stem cell-mediated immunosuppression occurs via concerted action of chemokines and nitric oxide. Cell Stem Cell, 2(2), 141-150.

63. William, T. T., Pendleton, J. D., Beyer, W. M., Egalka, M. C., \& Guinan, E. C. (2003). Suppression of allogeneic T-cell proliferation by human marrow stromal cells: implications in transplantation. Transplantation, 75(3), 389-397.

64. Ghannam, S., Pène, J., Torcy-Moquet, G., Jorgensen, C., \& Yssel, H. (2010). Mesenchymal stem cells inhibit human Th17 cell differentiation and function and induce a $\mathrm{T}$ regulatory cell phenotype. The Journal of Immunology, 185(1), 302-312.

65. Han, K. H., Ro, H., Hong, J. H., Lee, E. M., Cho, B., Yeom, H. J., Kim, M. G., Oh, K. H., Ahn, C., \& Yang, J. (2011).
Immunosuppressive mechanisms of embryonic stem cells and mesenchymal stem cells in alloimmune response. Transplant Immunology, 25(1), 7-15.

66. Comoli, P., Ginevri, F., Maccario, R., Avanzini, M. A., Marconi, M., Groff, A., Cometa, A., Cioni, M., Porretti, L., Barberi, W., \& Frassoni, F. (2008). Human mesenchymal stem cells inhibit antibody production induced in vitro by allostimulation. Nephrology Dialysis Transplantation, 23(4), 1196-1202.

67. Sotiropoulou, P. A., Perez, S. A., Gritzapis, A. D., Baxevanis, C. N., \& Papamichail, M. (2006). Interactions between human mesenchymal stem cells and natural killer cells. Stem Cells, 24(1), 74-85.

68. Xiao, J., Zhang, C., Zhang, Y., Zhang, X., Zhao, J., Liang, J., Zhong, X., \& Chen, Y. (2012). Transplantation of adiposederived mesenchymal stem cells into a murine model of passive chronic immune thrombocytopenia. Transfusion, 52(12), 25512558.

69. Bessout, R., Semont, A., Demarquay, C., Charcosset, A., Benderitter, M., \& Mathieu, N. (2014). Mesenchymal stem cell therapy induces glucocorticoid synthesis in colonic mucosa and suppresses radiation-activated T cells: new insights into MSC immunomodulation. Mucosal Immunology, 7(3), 656-669.

70. Karussis, D., Karageorgiou, C., Vaknin-Dembinsky, A., GowdaKurkalli, B., Gomori, J. M., Kassis, I., Bulte, J. W. M., Petrou, P., Benhur, T., Abramsky, O., \& Slavin, S. (2010). Safety and immunological effects of mesenchymal stem cell transplantation in patients with multiple sclerosis and amyotrophic lateral sclerosis. Archives of Neurology, 67(10), 1187-1194.

71. Lu, D., Chen, B., Liang, Z., Deng, W., Jiang, Y., Li, S., Xu, J., Wu, Q., Zhang, Z., Xie, B., \& Chen, S. (2011). Comparison of bone marrow mesenchymal stem cells with bone marrow-derived mononuclear cells for treatment of diabetic critical limb ischemia and foot ulcer: a double-blind, randomized, controlled trial. Diabetes Research and Clinical Practice, 92(1), 26-36.

72. Carrion, F., Nova, E., Ruiz, C., Diaz, F., Inostroza, C., Rojo, D., Mönckeberg, G., \& Figueroa, F. E. (2010). Autologous mesenchymal stem cell treatment increased $T$ regulatory cells with no effect on disease activity in two systemic lupus erythematosus patients. Lupus, 19(3), 317-322.

73. Chrzanowski, W., Kim, S. Y., \& McClements, L. (2020). Can stem cells beat COVID-19: Advancing stem cells and extracellular vesicles toward mainstream medicine for lung injuries associated with SARS-CoV-2 infections. Frontiers in Bioengineering and Biotechnology, 8, 554-561.

Publisher's Note Springer Nature remains neutral with regard to jurisdictional claims in published maps and institutional affiliations. 